\title{
Wind Characteristics and Wind Models for Wind Turbine Design in Japan (1st Report: Analysis of NEDO FT Data on Turbulence Characteristics and New Normal Turbulence Model Incorporating These Results).*
}

\author{
Tetsuya KOGAKI**, Hikaru MATSUMIYA ${ }^{* *}$, Hiroyuki ABE ${ }^{* *}$ \\ and Susumu OGAWA ${ }^{* * *}$ \\ ${ }^{* *}$ National Institute of Advanced Industrial Science and Technology (AIST), \\ 1-2-1, Namiki, Tsukuba, Ibaraki 305-8564, Japan \\ E-mail: kogaki.t@aist.go.jp \\ ***The Japan Electrical Manufacturers' Association (JEMA), \\ 17-4, Ichibancho, Chiyoda-ku, Tokyo 102-0082, Japan
}

\begin{abstract}
In order to survey the wind characteristics of Japan, where mountainous terrain is predominant, a large amount of wind data (NEDO Field Test Data) from more than 300 measurement sites in Japan were analyzed in detail, particularly the turbulence characteristics. As a result, it was found that the profiles of the $90 \%$ quantiles of turbulence intensity estimated in all of the NEDO Field Test Data are well approximated by the IEC Normal Turbulence Model (NTM). However, the turbulence intensity levels of more than $65 \%$ of the measurement datasets exceed the most severe turbulence category (Category A) in IEC 61400-1 (Ed.3). We therefore conclude that the present IEC turbulence model for wind turbine design does not reflect the severe wind conditions of mountainous countries such as Japan. This is considered to be one of the main reasons for the frequent problems of wind turbines in Japan. New criteria for the NTM are proposed to overcome this problem. The appropriateness of the new criteria to the severe wind characteristics in Japan is also discussed.
\end{abstract}

Key words: Wind Turbine, Wind Characteristics, Wind Models, Turbulence, Complex Terrain, Gust, Velocimetry

\section{Introduction}

The installed capacity of wind turbines in Japan exceeded 1,000 MW in 2006, and rapid further development is taking place to reach the goal of 3,000 MW by 2010. However, Japan's wind power development cannot necessarily be called a success from an international perspective. According to Ernst \& Young's Renewable Energy Country Attractiveness Indices ${ }^{(1)}$, Japan's short and long-term wind indices are not ranked (nor even considered candidates), indicating the unattractiveness of Japan as a destination for wind power development investments by the international market.

It is true that the rapid pace of wind power development worldwide can be partially

${ }^{*}$ Received 4 Sep., 2009 (No. T1-07-0219) Japanese Original : Trans. Jpn. Soc. Mech. Eng., Vol.73, No.733, B (2007), pp.1845-1852 (Received 15 Mar., 2007) [DOI: 10.1299/jee.4.467] 
attributed to incentives (particularly the Feed-in Tariff Law, amongst others) along with supportive policies such as the RPS Law. However, from a technological standpoint, continuing development of international standards for wind turbine generation technologies brought about by the IEC standards (IEC 61400 series) can be regarded as a major contributor. For example, wind turbines are classified according to extreme wind speeds and turbulent intensities in IEC $61400-1^{(2)}$ (Ed.3; JIS C 1400-1 is equivalent), a document that establishes safety requirements for wind power generation systems. This not only sets safe design standards, but also allows manufacturers to inexpensively mass-produce and distribute wind turbines around the world.

IEC 61400-1 is the fundamental document prescribing the international standards for wind turbine generation systems. As the primary concept for the safe design of wind turbine systems, it defines external parameters consisting of wind conditions, electrical power systems, and geographical conditions. The problem in the case of Japan is that its wind conditions are considered to be more severe than the international standards. This is due to the higher occurrence of turbulent winds and gusts created by the mountainous terrain, as well as the regular occurrence of typhoons. It is possible that the lack of consideration for Japan's severe wind conditions has been the cause of the problems surrounding imported wind turbines in recent years. More specifically, when comparing wind models for turbulence intensity and gusts as set forth in IEC 61400-1 with empirical data, the empirical data show far more severe wind characteristics ${ }^{(3)}$.

The purpose of this study is to develop wind models for wind turbine design that better reflect the wind characteristics of Japan, which are thought to be more severe than the European standards, so as to promote the smooth expansion of wind power generation capacity in Japan in the future. It can be assumed that other East Asian and Southeast Asian countries, in which a rapid expansion of wind power generation capacity is expected, have similar wind characteristics to Japan due to their topological and meteorological similarities. Therefore, wind models that properly reflect the wind characteristics of Japan may function as an universal standard for other Asian countries that are regularly subjected to typhoons and have turbulent winds and gusts due to complex terrains.

\section{Summary of NEDO FT data analyzed in this study}

This report focuses on analysis of turbulence intensity characteristics using wind data acquired by the Field Test Project on Wind Power Generation conducted by the New Energy and Industrial Technology Development Organization (NEDO). The field test data are referred to hereinafter as "NEDO FT Data." (4)

The NEDO FT Data used in this study consist of wind data collected at 304 locations between 1995 and 2002. Many of the measurements in the NEDO Field Test Project were taken at locations known to have favorable wind conditions, such as in the Hokkaido, Tohoku, Kyushu, and Okinawa regions, although overall, the locations were scattered throughout the country. Therefore, the data used in this report should properly reflect the wind characteristics of sites suitable for wind power generation in Japan.

The NEDO FT Data include the 1-hour average and 10-minite average of wind speed, direction and turbulence standard deviation. The measurements were taken over a period of 1 year, at measurement heights of 10,20 , or $30 \mathrm{~m}$. In some years, measurements were taken at multiple heights (heights above ground of 10 and $20 \mathrm{~m}$, or 20 and $30 \mathrm{~m}$ ), while in other years measurements were taken only at a single height (height above ground of $20 \mathrm{~m}$ only). If the wind data obtained over 1 year at a given location and a height are considered to be 1 dataset, a total of 523 datasets were taken into consideration for the analysis in this study. Since the companies and institutions that collect the NEDO FT Data are planning to conduct wind power generation, it is assumed that personnel collecting the data are knowledgeable 
and take measurements appropriately. However, perhaps due to inexperience in taking wind measurements, there were several instances where the required data were missing (4 datasets). Some erratic data, assumed to be due to inept measurements, were also observed ( 2 datasets). Additionally, perhaps due to noise in data, there were cases where data showed unnatural or abnormally concentrated distribution ( 5 datasets). Consequently, a total of 512 datasets were actually used in this analysis after excluding the above-mentioned 11 abnormal datasets.

In almost all cases, NEDO FT Data acquisition utilizes a measurement mast, cup anemometer, and wind vane from NRG Systems. Although cup-type anemometers may be sufficiently reliable for the purpose of business risk assessment by wind power producers, or for prediction of annual energy production, it is doubtful whether they are appropriate for reliable turbulence analysis and assessment as desired in this study. We therefore verified the validity of turbulence intensity in the NEDO FT Data through separate actual measurements. The findings from these separate measurements will be discussed in the 2 nd report.

\section{Turbulence analysis results of NEDO FT data}

\subsection{Typical turbulence characteristics in Japan}

In order to understand the typical turbulence intensity characteristics of Japan's wind power generation sites, a $90 \%$ quantile distribution of turbulence intensity was obtained by averaging all datasets of the NEDO FT Data. In this study, a binning method was used for the $90 \%$ quantile calculations, but the instructions for calculating the $90 \%$ quantile as outlined in IEC 61400-1 (Ed.3) are vague and do not provide sufficient detail. In this report, normal distribution is assumed for calculation of the $90 \%$ quantile. Any effect on the analysis results due to the difference in method of $90 \%$ quantile calculation is discussed in Appendix A. An averaged $90 \%$ quantile distribution was calculated by weighted-averaging according to the number of valid data point in each bin in each dataset. This is equivalent to determining the $90 \%$ quantile of turbulence intensity of the all valid NEDO FT Data. Here, the width of the wind speed bin was set to $1 \mathrm{~m} / \mathrm{s}$. Figure 1 shows the $90 \%$ quantile distribution of turbulence intensity after averaging the NEDO FT Data, using the above-mentioned method.

The averaged $90 \%$ quantile of turbulence intensity of NEDO FT Data is considered to reflect the turbulence intensity characteristics of the most representative sites suitable for wind power generation in Japan. It can be seen that the overall distribution of the $90 \%$ quantile of turbulence intensity is very similar to Category A (high-turbulence case, $I_{\text {ref }}=$ $0.16, I_{\text {ref }}$ : expected value of turbulence intensity, $\left.T I\right)$ of the Normal Turbulence Model (NTM) in IEC 61400-1 (Ed.3), except that it is slightly smaller for low wind speeds below 6 $\mathrm{m} / \mathrm{s}$. Moreover, the value of $I_{r e f}$ is 0.159 , which is in close agreement with the value of $I_{r e f}$ in Category A. The latest large-scale wind turbines in Europe are often designed with Category $\mathrm{B}$ in mind (moderate-turbulence case, $I_{r e f}=0.14$ ). Therefore, even though the average turbulence intensity of Japan is in Category A of the IEC NTM standard, it is potentially above the design criteria of mainstream large-scale wind turbines in Europe. 


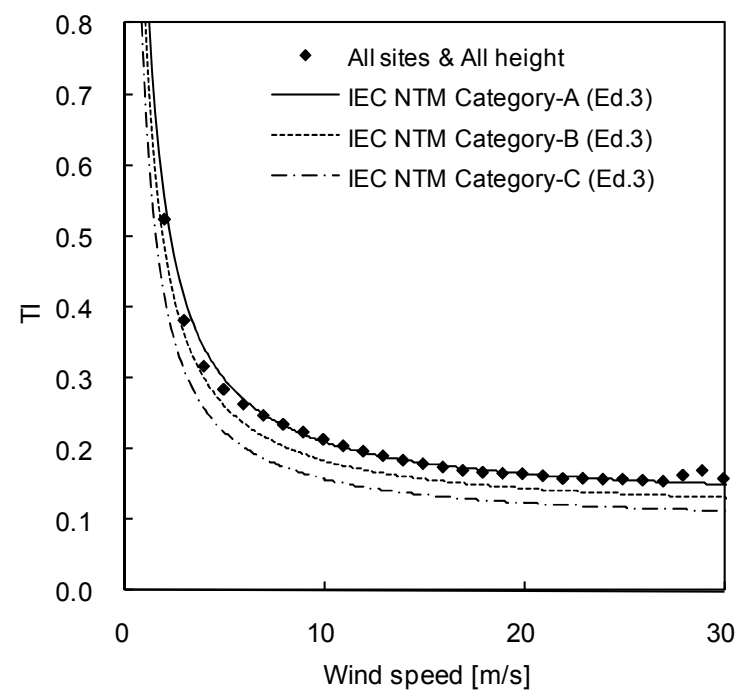

Fig. $190 \%$ quantiles of turbulence intensity of all NEDO FT datasets as a function of 10-minute average wind speed.

\subsection{Turbulence intensity characteristics of individual sites}

For all valid data from the NEDO FT Data, the $90 \%$ quantile distributions of turbulence intensity for average wind speed and for individual sites were graphed. Since the number of datasets is very high at 512, the resulting graphs were too many and were omitted from this report. Also, $I_{\text {ref }}$ was obtained for a wind speed of $15 \mathrm{~m} / \mathrm{s}$. The parameter $I_{\text {ref }}$ for this report was obtained from the IEC NTM model equation, as follows:

$$
T I=\frac{I_{\text {ref }}\left(0.75 \times V_{h u b}+b\right)}{V_{h u b}},
$$

where,

$T I$ : turbulence intensity, $T I=\operatorname{Std}(V) / V_{\text {hub }}$;

$\operatorname{Std}(V)$ : standard deviation of wind speed $V$, for duration of 10 minutes;

$V_{h u b}:$ 10-minite average wind speed at hub height;

$b$ : constant, $5.6[\mathrm{~m} / \mathrm{s}]$;

with $V_{h u b}=15[\mathrm{~m} / \mathrm{s}]$ substituted, thus giving

$I_{\text {ref }}=T I / 1.123$,

as the final equation for $I_{r e f}$.

The similarities between the $90 \%$ quantile distribution of each dataset and the IEC NTM were compared. Their similarities (profile shape and intensity level) were ranked as "high," "moderate," or "low." The comparison results can be classified into five cases, as follows:

(a) Both the profile shape and intensity level are in close agreement with the IEC NTM, and thus the IEC NTM should be adequate to model the specific case (the profile shape and intensity level similarities are both ranked "high").

(b) The profile shape is similar, but the intensity level does not correspond (the profile shape similarity is ranked "high" but the intensity level similarity is ranked "moderate" or "low").

(c) The intensity level is similar, but the profile shape is different (the intensity level similarity is ranked "high" but the profile shape similarity is ranked "moderate").

(d) Both the profile shape and intensity level are somewhat different from the IEC NTM (the profile shape and intensity level similarities are both ranked "moderate").

(e) The profile shape and/or intensity level are distinctly different (either one or both 
of the profile shape and intensity level similarities have "low" ranking, except for the case the profile shape similarity is ranked "high").

Although the analysts conducted classification subjectively, decisions were based on the extent and range of differences and were made as objectively as possible. The results of the classification are shown in Table 1 . The table indicates that for more than $90 \%$ of the datasets (467 datasets out of 512), the profile shapes show some similarity to the IEC NTM (the profile shape similarity is ranked "moderate" or higher; cases (a), (b), (c), and (d)). Hence, the profile shapes of the IEC NTM adequately represent the turbulence intensity characteristics seen in Japan. Specifically, about $40 \%$ also have a high similarity in intensity level (cases (a) and (c); 202 datasets). Therefore, the IEC NTM should be adequate for these cases as well. On the other hand, most of the cases that were ranked "moderate" for intensity level similarity (cases (b) and (d); 265 datasets out of 512), have a $90 \%$ quantile distribution larger than the IEC NTM, and in many cases, $I_{\text {ref }}$ exceeds 0.20 . Thus, the value of $I_{\text {ref, }}$ an equation parameter in the IEC NTM, does not adequately reflect the wind characteristics of Japan and, at many sites, the turbulence intensity actually exceeds IEC Category A (high-turbulence category).

Table 1 Comparison of similarities between profiles of $90 \%$ quantiles of turbulence intensities and the IEC NTM.

\begin{tabular}{|l|l|l|l|r|}
\hline Case & Classification & \multicolumn{2}{|c|}{ Similarity } & $\begin{array}{l}\text { Number of } \\
\text { datas ets }\end{array}$ \\
\cline { 3 - 5 } a & $\begin{array}{l}\text { Both the profile shape and the intensity } \\
\text { level agree well with IEC NTM. }\end{array}$ & High & High & 105 \\
\hline b & $\begin{array}{l}\text { The profile shape agrees well with IEC } \\
\text { NTM, but the intensity level somewhat } \\
\text { differs from IEC NTM. }\end{array}$ & High & $\begin{array}{l}\text { Moderate or } \\
\text { Low }\end{array}$ & 58 \\
\hline c & $\begin{array}{l}\text { The intensity level agrees well with IEC } \\
\text { NTM, but the profile shape somewhat } \\
\text { differs from IEC NTM. }\end{array}$ & Moderate & High & 97 \\
\hline d & $\begin{array}{l}\text { Both the profile shape and the itensity } \\
\text { level somewhat differ from IEC NTM. }\end{array}$ & Moderate & Moderate & 207 \\
\hline e & $\begin{array}{l}\text { Both the profile shape and the itensity } \\
\text { level considerably differ from IEC NTM. }\end{array}$ & $\begin{array}{l}\text { Moderate and Low, Low and } \\
\text { Moderate or Low and Low }\end{array}$ \\
\hline Total & \multicolumn{2}{|l}{} & 45 \\
\hline
\end{tabular}

\subsection{Changes in turbulence intensity according to measurement height}

Figure 2 shows the averaged 90\% quantile distribution of turbulence intensity at different measurement heights. The average values were calculated by the similar method as described in Section 3.1. It is seen that turbulence intensity increases slightly at lower measurement heights. However, since Fig. 2 was obtained using all of the datasets, it is possible that differences in measurement sites may have influenced the results. Thus, Fig. 3 shows the average of the datasets, taken only from sites where measurements were recorded at both heights. The basic trend of turbulence intensity decreasing as the height from the ground increases is still seen in Fig. 3.

The present IEC NTM does not include the measurement height above ground (or height of wind turbine hub, $Z_{h u b}$ ) as a parameter in the model equations. This parameter serves as an added safety factor for large wind turbines with a hub at a high elevation, but represents an added risk for smaller wind turbines that have been receiving increased attention for practicality and safety. 


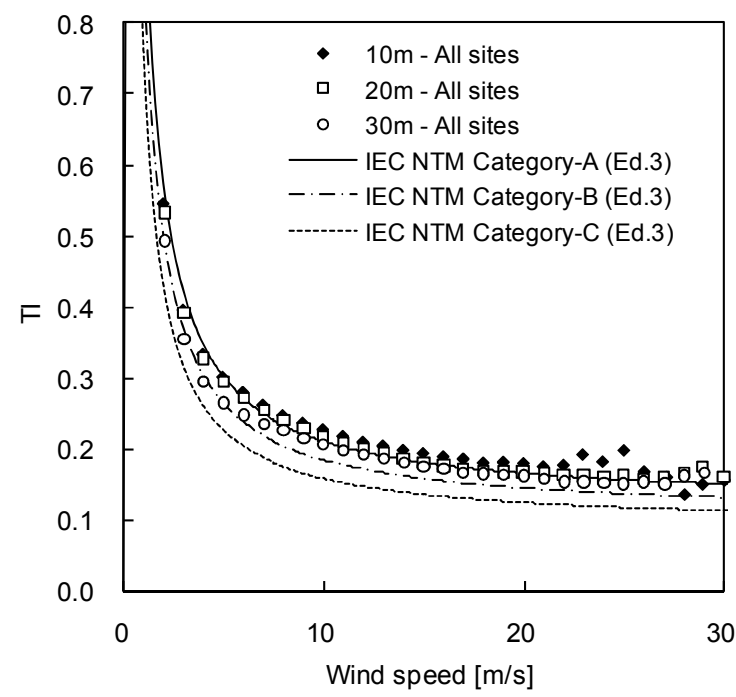

Fig. $290 \%$ quantiles of turbulence intensity evaluated at each height as a function of 10-minute average wind speed (all NEDO FT datasets).

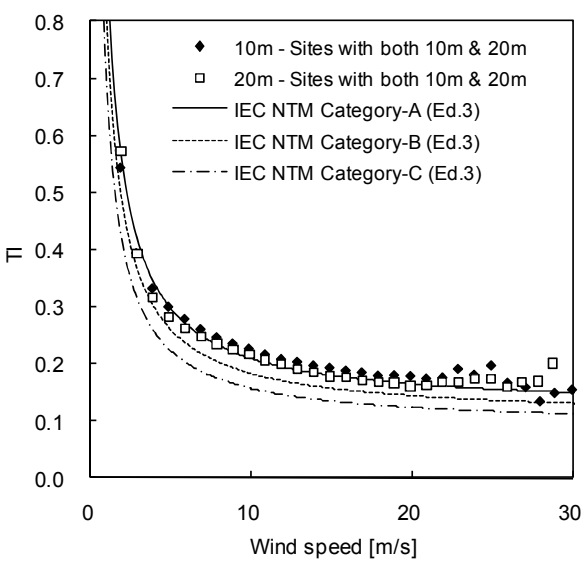

(a) $10[\mathrm{~m}]$ and $20[\mathrm{~m}]$.

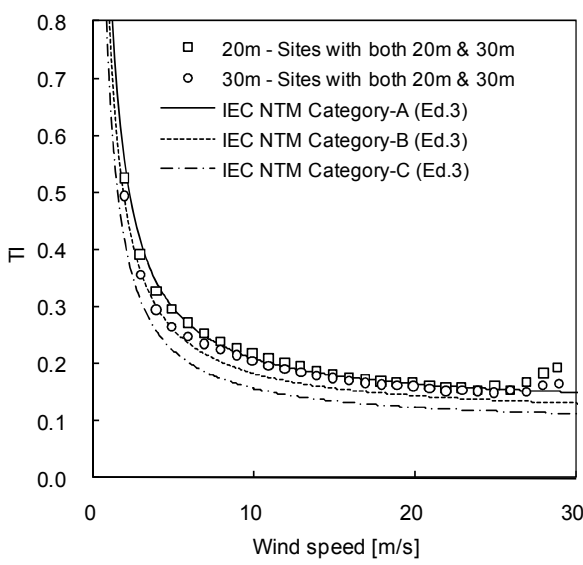

(b) $20[\mathrm{~m}]$ and $30[\mathrm{~m}]$.

Fig. $390 \%$ quantiles of turbulence intensity evaluated at each height as a function of 10 -minute average wind speed (only sites where measurement was conducted at plural heights).

\section{Wind model reflecting wind characteristics in Japan}

It may be possible to derive model equations with height as a parameter, or to derive parameters themselves for flat landforms based on a physical hypothesis. However, due to the intricacies of the phenomenon, it is almost impossible to derive model equations or parameters for complex geometries based on the basis of a physical hypothesis alone, such as discussed in this report. Therefore, we will derive model equations based on empirical measurements. As mentioned in Section 3, the current IEC NTM functionally approximates turbulence intensity distribution. Therefore, the derivation of a turbulence model that reflects the wind characteristics of Japan can use the current IEC NTM model as a basis, with parameters adjusted to match the turbulence intensity levels of Japan.

\subsection{Consideration of effect of height above ground}

In Section 3.3, it was confirmed that turbulence intensity is affected by the height above 
ground, and that the turbulence intensity level decreases as the height above ground increases. As a result, we believe that a turbulence model needs to consider the height above ground, and thus propose a turbulence intensity model that takes this into consideration.

As already mentioned, the parameter $I_{r e f}$ specifies turbulence intensity in the IEC NTM. $I_{\text {ref }}$ is also a parameter in other wind models (e.g., the Extreme Operating Gust (EOG) model, Extreme Turbulence Model (ETM), etc.) in IEC 61400-1 (Ed.3) ${ }^{(2)}$. In this report, we propose that $I_{\text {ref }}$ be a function of the height above ground, instead of a constant specific to each category as treated by the IEC NTM.

Figure 4 shows $I_{r e f}$ as a function of the height above ground. The change in $I_{r e f}$ as a function of the height above ground, $z$, can be approximated as follows:

$$
I_{\text {ref }}=0.2149 z^{-0.0951} \text {. }
$$

Thus, $I_{\text {ref }}$ decreases with respect to the height above ground to the power of -0.0951 . If we ignore the absolute value of the approximate equation, and make the assumption that $I_{r e f}$ in the current IEC NTM was designed to accommodate a hub height of $60 \mathrm{~m}$ (typical for large wind turbines prevalent in Europe), the model equations that take the change in height into consideration can be expressed as follows:

Category A, high-turbulence case:

$$
I_{\text {ref }}=0.16\left(\frac{z}{z_{b}}\right)^{-0.0951}
$$

Category B, moderate-turbulence case:

$$
I_{r e f}=0.14\left(\frac{z}{z_{b}}\right)^{-0.0951}
$$

Category C, low-turbulence case:

$$
I_{\text {ref }}=0.12\left(\frac{z}{z_{b}}\right)^{-0.0951},
$$

where,

$z_{b}: 60[\mathrm{~m}]$.

The constant on the right-hand side of each of the above equations is $I_{\text {ref }}$ in the current IEC turbulence model. Figure 5 shows a comparison between the new $I_{\text {ref }}$, as derived above, and the results of analysis of the NEDO FT Data. Since the height above ground was taken into consideration, the typical turbulence intensity in Japan is now only slightly above that of Category B.

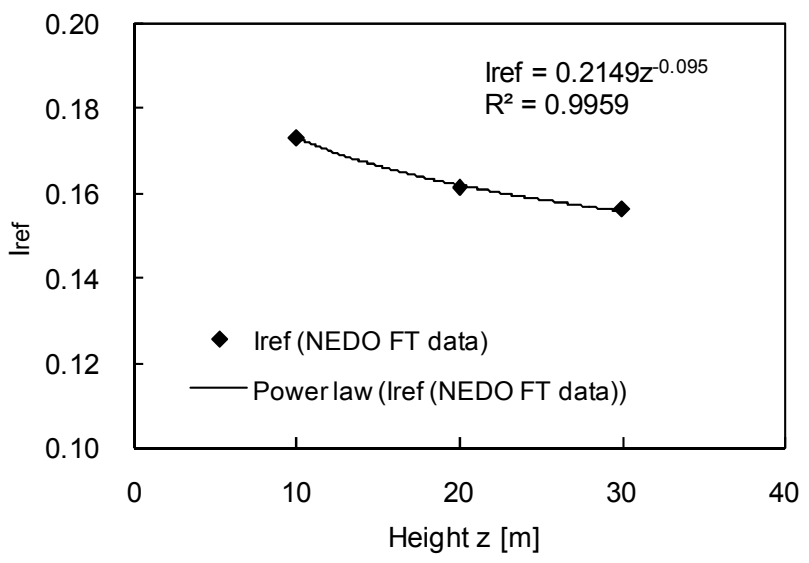

Fig. $4 I_{r e f}$ as a function of height $z$. 


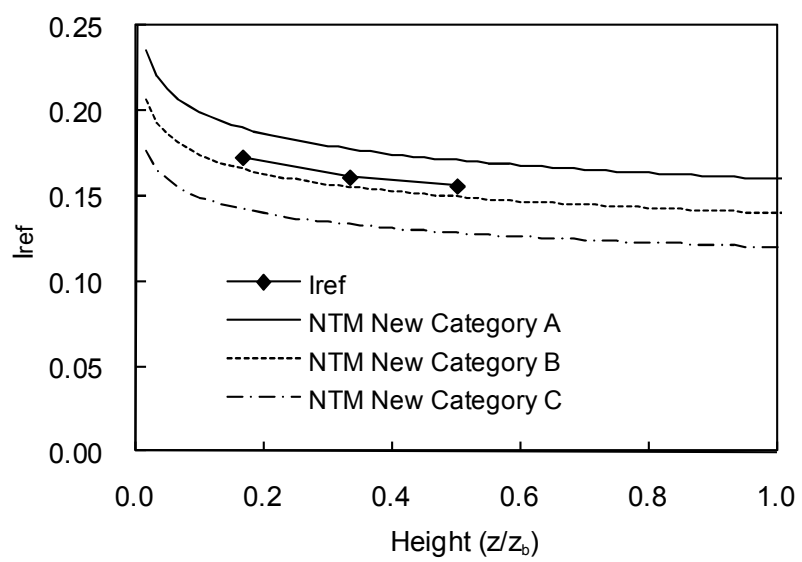

Fig. $5 I_{\text {ref }}$ as a function of height $\mathrm{z}$.

\subsection{Consideration of turbulence intensity level}

Figure 6 shows a histogram of $I_{\text {ref }}$ (including all datasets), while Table 2 shows the detailed distribution of the same parameter. Note that in this figure and table, \#N/A denotes cases where evaluation of $I_{\text {ref }}$ was not possible due to insufficient or nonexistent data at a wind speed of $15 \mathrm{~m} / \mathrm{s}$. In the figure and table, based on the current IEC 61400-1 (Ed.3) and considering all datasets, $6.0 \%$ would be classified as Category C (low-turbulence case) or below, $14.4 \%$ would be classified as Category B (moderate-turbulence case) or below, and only $31.8 \%$ would be classified as Category A (high-turbulence case) or below. This leaves an exorbitant $68.2 \%$ of datasets classified as Class S. As a result, it is clear that the current IEC NTM is insufficient for modeling the wind characteristics of Japan, as Japanese turbulence characteristics far exceed the IEC criterion. We recognize that wind turbine manufacturers prefer to supply wind turbines based on one set of design criteria as a cost-cutting measure. Furthermore, it is relatively rare for manufacturers to design and develop Class $\mathrm{S}$ wind turbines, which require specific design and development for individual sites. There is also a danger that wind power project developer or wind power producers would reluctantly procure Category B wind turbines due to limited availability of Class $\mathrm{S}$ wind turbines, even though they realized that a particular wind power generation site exhibited wind characteristics for which a Class $\mathrm{S}$ design was appropriate. It is conceivable that these current circumstances are a factor contributing to the wind turbine problems seen in Japan in recent years. If a new official category were to be created covering the majority of sites in Japan, wind turbines designed to comply with this category could operate normally in almost all areas of the country, without frequent problems, and without increased risk to power generators. This, it is hoped, would greatly improve the current situation. 


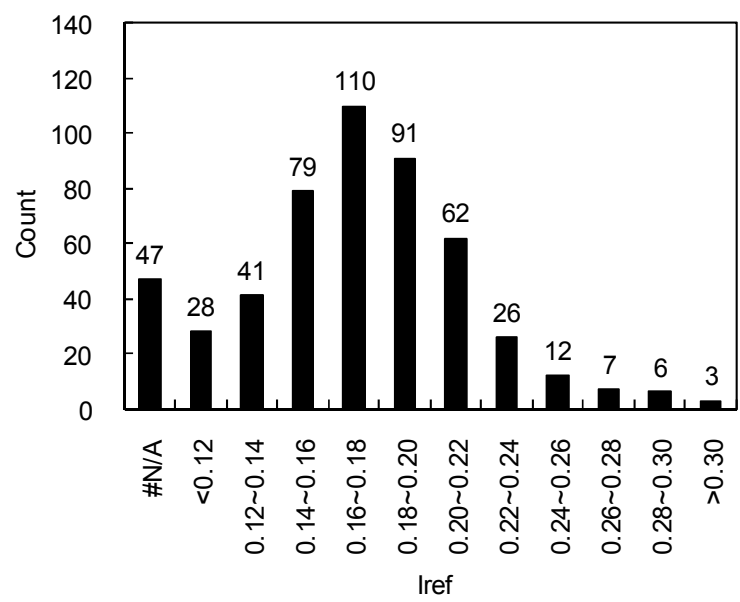

Fig. $6 I_{\text {ref }}$ as a function of height $z$.

Table 2 Classification of $I_{r e f}$.

\begin{tabular}{|c|c|c|c|c|c|c|c|c|c|c|c|c|}
\hline \multirow{2}{*}{ Iref } & \multicolumn{3}{|l|}{$10[\mathrm{~m}]$} & \multicolumn{3}{|l|}{20 [m] } & \multicolumn{3}{|l|}{$30[\mathrm{~m}]$} & \multicolumn{3}{|l|}{ all } \\
\hline & \begin{tabular}{|l|} 
Count \\
\end{tabular} & Ratio & Cumu. & Count & Ratio & Cumu. & Count & Ratio & Cumu. & Count & Ratio & Cumu. \\
\hline \#NA & 11 & & & 25 & & & 11 & & & 47 & & \\
\hline$<0.12$ & 1 & $3.4 \%$ & $3.4 \%$ & 14 & $5.1 \%$ & $5.1 \%$ & 13 & $7.9 \%$ & $7.9 \%$ & 28 & $6.0 \%$ & $6.0 \%$ \\
\hline $0.12 \sim 0.14$ & 2 & $6.9 \%$ & $10.3 \%$ & 24 & $8.8 \%$ & $14.0 \%$ & 15 & $9.1 \%$ & $17.1 \%$ & 41 & $8.8 \%$ & $14.8 \%$ \\
\hline $0.14 \sim 0.16$ & 5 & $17.2 \%$ & $27.6 \%$ & 42 & $15.4 \%$ & $29.4 \%$ & 32 & $19.5 \%$ & $36.6 \%$ & 79 & $17.0 \%$ & $31.8 \%$ \\
\hline $0.16 \sim 0.18$ & 9 & $31.0 \%$ & $58.6 \%$ & 56 & $20.6 \%$ & $50.0 \%$ & 45 & $27.4 \%$ & $64.0 \%$ & 110 & $23.7 \%$ & $55.5 \%$ \\
\hline $0.18 \sim 0.20$ & 3 & $10.3 \%$ & $69.0 \%$ & 65 & $23.9 \%$ & $73.9 \%$ & 23 & $14.0 \%$ & $78.0 \%$ & 91 & $19.6 \%$ & $75.1 \%$ \\
\hline $0.20 \sim 0.22$ & 4 & $13.8 \%$ & $82.8 \%$ & 35 & $12.9 \%$ & $86.8 \%$ & 23 & $14.0 \%$ & $92.1 \%$ & 62 & $13.3 \%$ & $88.4 \%$ \\
\hline $0.22 \sim 0.24$ & 3 & $10.3 \%$ & $93.1 \%$ & 18 & $6.6 \%$ & $93.4 \%$ & 5 & $3.0 \%$ & $95.1 \%$ & 26 & $5.6 \%$ & $94.0 \%$ \\
\hline $0.24 \sim 0.26$ & 0 & $0.0 \%$ & $93.1 \%$ & 5 & $1.8 \%$ & $95.2 \%$ & 7 & $4.3 \%$ & $99.4 \%$ & 12 & $2.6 \%$ & $96.6 \%$ \\
\hline $0.26 \sim 0.28$ & 0 & $0.0 \%$ & $93.1 \%$ & 7 & $2.6 \%$ & \begin{tabular}{|l|}
$97.8 \%$ \\
\end{tabular} & 0 & \begin{tabular}{|l|l|}
$0.0 \%$ \\
\end{tabular} & $99.4 \%$ & 7 & $1.5 \%$ & $98.1 \%$ \\
\hline $0.28 \sim 0.30$ & 2 & $6.9 \%$ & $100.0 \%$ & 4 & $1.5 \%$ & $99.3 \%$ & 0 & $0.0 \%$ & $99.4 \%$ & 6 & $1.3 \%$ & $99.4 \%$ \\
\hline$>0.30$ & 0 & $0.0 \%$ & $100.0 \%$ & 2 & $0.7 \%$ & $100.0 \%$ & 1 & $0.6 \%$ & $100.0 \%$ & 3 & $0.6 \%$ & $100.0 \%$ \\
\hline Sum & 40 & $100.0 \%$ & & 297 & $100.0 \%$ & & 175 & $100.0 \%$ & & 512 & $100.0 \%$ & \\
\hline
\end{tabular}

Table 3 shows measured $I_{r e f}$ sorted based on modified $I_{r e f}$ values that consider the height above ground, as derived in Section 4.1. Even with the new turbulence model equation, the tabulated results indicate that approximately 30 to $50 \%$ of the datasets would be classified as Class S. Therefore, it is clear that a new category needs to be established to reflect the severe wind characteristics of Japan. After careful review of the data summarized in Tables 2 and 3, we propose the following new categories:

Category J1, complex-terrain, high-turbulence case:

$$
I_{\text {ref }}=0.20\left(\frac{z}{z_{b}}\right)^{-0.0951},
$$

Category J2, complex-terrain, low-turbulence case:

$$
I_{\text {ref }}=0.18\left(\frac{z}{z_{b}}\right)^{-0.0951} .
$$

A comparison between the new turbulence model, existing conventional IEC turbulence model, and measured values ( $90 \%$ quantile) is shown in Fig. 7. As seen in the figures, using the new turbulence model, the typical turbulence intensity of various sites around Japan at heights above ground of 10,20 , and $30 \mathrm{~m}$ would generally be classified as Category B (flat-terrain, moderate-turbulence case). In the new turbulence model, $I_{\text {ref }}$ changes with the height above ground, becoming smaller as the height above ground increases. The new 
model matches the conventional IEC turbulence model at a height above ground of $60 \mathrm{~m}$.

Table 4 shows measured $I_{r e f}$ classified according to the new turbulence model. With the new model, approximately $90 \%$ of the turbulence intensity as observed in the NEDO FT datasets is in Category J1. Furthermore, approximately $75 \%$ is in Category J2, and approximately $50 \%$ is in Category A. Only a small quantity of approximately $10 \%$ exceeds Category J1, thus falling into Class S. At this point, special design for a high-turbulence-intensity site would be appropriate.

Table 3 Classification of turbulence category of All NEDO FT datasets.

\begin{tabular}{|l|r|r|r|}
\hline Category & \multicolumn{1}{l|}{ Height } & \multicolumn{1}{l|}{ 10 [m] } & \multicolumn{1}{l|}{$30[\mathrm{~m}]$} \\
\hline Category S & $31.0 \%$ & $51.8 \%$ & $47.6 \%$ \\
\hline Category A & $69.0 \%$ & $48.2 \%$ & $52.4 \%$ \\
\hline Category B & $34.5 \%$ & $26.8 \%$ & $28.7 \%$ \\
\hline Category C & $13.8 \%$ & $9.2 \%$ & $11.6 \%$ \\
\hline
\end{tabular}

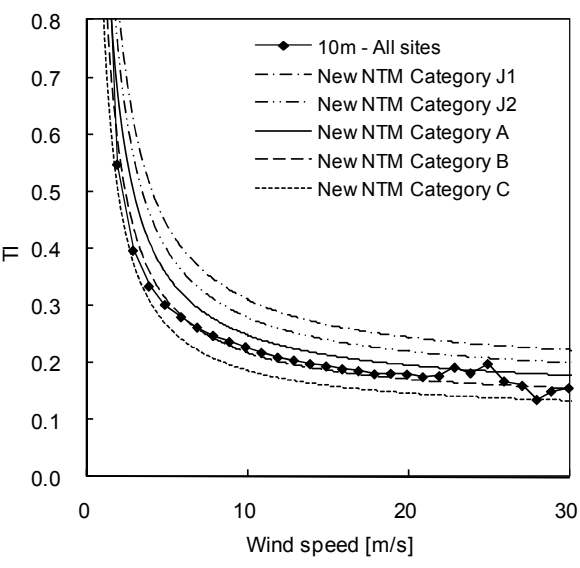

(a) $10 \mathrm{~m}$.

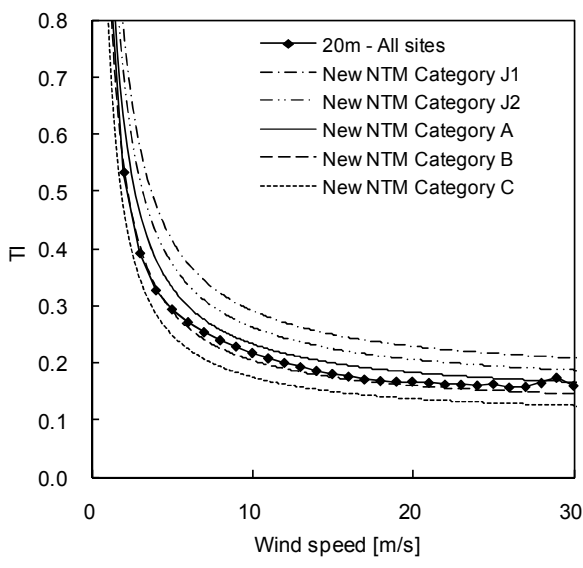

(b) $20 \mathrm{~m}$.

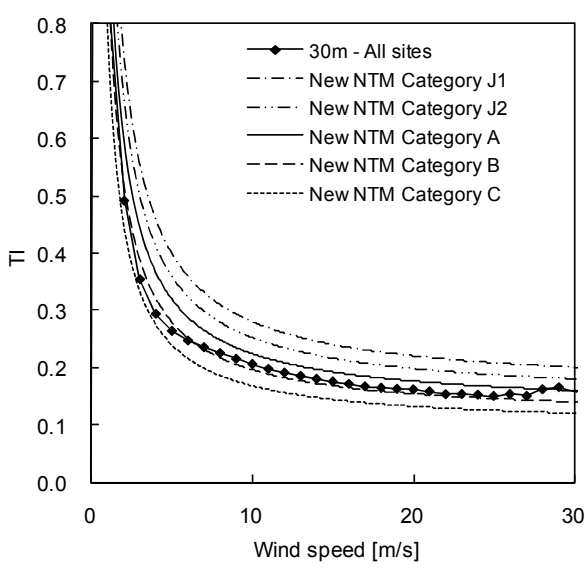

(c) $30 \mathrm{~m}$.

Fig. $7 \quad 90 \%$ quantiles of turbulence intensity as a function of 10 -minute average wind speed. 
Table 4 Classification of turbulence categories by $I_{\text {ref }}$

\begin{tabular}{|l|l|l|l|}
\hline Category & \multirow{2}{|c|}{ Height } & $20[\mathrm{~m}]$ & $30[\mathrm{~m}]$ \\
\hline Category S & $6.9 \%$ & $12.9 \%$ & $11.6 \%$ \\
\hline Category J1 & $13.8 \%(93.1 \%)$ & $13.2 \%(87.1 \%)$ & $14.6 \%(88.4 \%)$ \\
\hline Category J2 & $10.3 \%(79.3 \%)$ & $25.7 \%(73.9 \%)$ & $21.3 \%(73.8 \%)$ \\
\hline Category A & $34.5 \%(69.0 \%)$ & $21.3 \%(48.2 \%)$ & $23.8 \%(52.4 \%)$ \\
\hline Category B & $20.7 \%(34.5 \%)$ & $17.6 \%(26.8 \%)$ & $17.1 \%(28.7 \%)$ \\
\hline Category C & $13.8 \%$ & $9.2 \%$ & $11.6 \%$ \\
\hline
\end{tabular}

\section{Conclusion}

Through analysis of turbulence characteristics in Japan using NEDO FT Data, which are considered to cover most sites that are suitable for wind power generation in Japan, we obtained the following findings:

(1) Typical turbulence intensity distributions in Japan are very similar to that of Category A, the high-turbulence case in the current IEC 61400-1 (Ed.3).

(2) By comparing turbulence intensity levels at various heights above ground, it was found that the turbulence intensity decreases with increasing height above ground, and that the rate of change in turbulence intensity level corresponds to the height above ground to the power of -0.0951 .

(3) Based on the above, a new model was developed and proposed for each category of turbulence intensity. The new model has varying values of $I_{\text {ref }}$ to properly reflect the turbulence intensity.

(4) It was noted that more than $65 \%$ of the datasets of the NEDO FT Data would be classified as Class $\mathrm{S}$, which is beyond the most severe turbulence classification of Category $\mathrm{A}$ in the current IEC standard.

(5) We proposed two additional categories to cope with the more severe turbulence intensity levels encountered across Japan. Then, using these two new categories, we verified that approximately $90 \%$ of datasets of the NEDO FT Data would fall within valid turbulence categories.

It is important to note that compatibility between a NTM that uses $I_{\text {ref }}$ varying with the height above ground, as proposed in this report, and other IEC wind models, is not yet fully understood. As a result, further investigation is required. Furthermore, since the parameters in the model proposed in this report were set based solely on heights above ground of 10, 20 , and $30 \mathrm{~m}$, an understanding of reliability at higher heights above ground is lacking. Further analysis, including measured data from increased heights above the ground, is required.

\section{Acknowledgement}

Part of the research for this report was conducted while the first author was dispatched overseas as a researcher through the Japan Society for the Promotion of Science Postdoctoral Fellowship for Research Abroad program (dispatched from February 2005 to February 2007).

\section{Appendix A. Method for calculating 90\% quantile of turbulence intensity}

A detailed method of calculating the $90 \%$ quantile of turbulence intensity is not clearly specified in IEC 61400-1 (Ed.3). It is possible that discrepancies in the method of 
calculating the $90 \%$ quantile may lead to differences in turbulence characteristics analysis results. Based on an assumed distribution of turbulence intensity in the wind speed bin, there are three possible methods of calculating the $90 \%$ quantile:

1) Calculate it directly without assuming a specific distribution.

2) Assume normal distribution.

3) Assume lognormal distribution.

Method 2) was selected here, with the following equation used to calculate the $90 \%$ quantile:

$$
\sigma_{90 q}=E(\sigma)+1.28 \operatorname{Std}(\sigma)
$$

where

$\sigma_{90 q}: 90 \%$ quantile of standard deviation of wind speed $V$;

$\sigma:$ standard deviation of wind speed $V, \sigma=\operatorname{Std}(V)$;

$E(X)$ : average of a random variable $X$;

$\operatorname{Std}(X)$ : standard deviation of a random variable $X$.

Since a statement pertaining to Eq. (A-1) is referenced in IEC 61400-1 (Ed.3), it can be interpreted that IEC assumes a normal distribution. On the other hand, since the actual $\sigma$ is the random variable with $\sigma>0$, the assumption of a lognormal distribution would be considered more natural. Furthermore, a passage that assumes lognormal distribution is found in IEC 61400-1 Ed.3 (p. 25, Note 4). These two statements in the IEC seem contradictory. However, for a realistic standard deviation $\sigma$ that would be encountered in actual wind speed data, "a method assuming normal distribution" is almost equivalent to "a method assuming lognormal distribution."

Meanwhile, it is expected that as the measurement duration lengthens and the number of data points increase in each wind speed bin, the differences between each of the distributions will become relatively small. In other words, the difference between method 1), directly calculating quantiles without assuming a specific distribution, and methods 2) and 3), assuming either normal distribution or lognormal distribution, will diminish so that "method 1)" is roughly equivalent to "method 2)" or "method 3)." However, it is important to clarify that this expected behavior is for the turbulence characteristics of flat terrain, with no guarantee that turbulence characteristics in complex terrain would assume either normal or lognormal distribution. An investigation of the repercussions of the above on the results of analysis using NEDO FT Data shows that in some cases, in isolation, significant differences can exist depending on the assumption of distribution. However, from a statistical point of view, it was verified that differences between the results of method 1) and method 2) are insignificant. Therefore, for the purpose of the analysis results and discussion presented here, we judge that it is sufficient to assume that method 1) is almost equivalent to methods 2) and 3).

\section{References}

(1) Ernst \& Young, Renewable Energy Country Attractiveness Indices - 2006 Winter, http://www.ey.com

(2) International Electrotechnical Commission, IEC 61400-1 Ed.3, Wind turbines - Part 1: Design requirements, 2005.

(3) Kogaki, T, Matsumiya, H. and Ogawa, S., A Conception for Development of the J Class Wind Model, Proceedings of the 25th Wind Energy Utilization Symposium, pp. 221-224, 2003 (in Japanese).

(4) New Energy and Industrial Technology Development Organization (NEDO), Database on technical information, http://www.nedo.go.jp/database/index.html. 